\title{
PRODUITS FINANCIERS ET DETERMINATION DE LA PRIME GLISSANTE DE TRAITES NON PROPORTIONNELS
}

\author{
By CHARLES LeVI \\ Compagnie Transcontinentale de Reassurance, Paris, France
}

\begin{abstract}
RESUME
Les produits financiers jouent un rôle important dans le calcul de l'étendue de la fourchette d'une prime glissante de traités non-proportionnels. Le sinistre total à charge de l'XL est représenté par un processus composé de Poisson. Il est calculé à l'aide de l'algorithme de Panjer. Sont ensuite présentés des résultats pour différentes valeurs du nombre de sinistres à charge et du taux de rendement financier.
\end{abstract}

\begin{abstract}
Financial income play a big part for the calculation of the minimum premium and maximum premium of a sliding scale for non-proportional treaties. The aggregate loss for the $\mathrm{XL}$ is supposed to be a compound Poisson process. It is computed by Panjer's Algorithm. Some numerical results are shown for different values of the claims number and the interest rate.
\end{abstract}

\section{KEYWORDS}

Rating of XL treaties, sliding scale, financial income

La prime pour des traités de réassurance non-proportionnels se présente généralement sous une des trois formes principales suivantes:

-une prime fixe définie par un taux de prime appliqué à un encaissement défini dans le texte contractuel du traité.

-une prime flat, i.e. un montant monétaire fixe défini à priori.

-une prime variable ou glissante. Trois paramètres définissent le taux de prime associé que l'on appellera taux de prime glissant par la suite: un taux minimum, un taux maximum et un taux de chargement.

soit $x_{0}$ : priorité du traité non-proportionnel

$k x_{0}:$ plafond du traité non-proportionnel

$x_{1}, x_{2} \ldots$ sinistres individuels

$P$ : encaissement de prime défini dans le texte contractuel du traité

$t_{m}:$ taux de prime minimum

$t_{M}:$ taux de prime maximum

$t$ : taux de chargement 
$b c$ : burning cost

$t_{\mathrm{g}}$ : taux de prime glissant

(1) l'on a: $b c=\sum_{i} \frac{\operatorname{Min}\left(\operatorname{Max}\left(x_{i}-x_{0}, 0\right), k x_{0}-x_{0}\right)}{P}$

$$
t_{\mathrm{g}}=\operatorname{Min}\left(\operatorname{Max}\left(t_{m}, \frac{b c}{1-t}\right), t_{\mathrm{M}}\right)
$$

Dans les branches dans lesquelles le déroulement des sinistres s'effectue rapidement, la période de temps s'écoulant entre le versement de la prime (qui s'appuie sur une estimation des sinistres) et le paiement effectif des sinistres est réduite.

En conséquence, le rôle que peuvent jouer les revenus financiers demeure de peu d'importance. En revanche dans des branches 'long tail' (à déroulement long) comme la RC Auto ou la RC générale, cette période peut s'étendre sur plusieurs années. L'influence des revenus financiers sur les résultats du traité apparaissent ainsi de façon très nette.

L'étude ci-dessous porte sur le calcul des conditions d'un taux de prime glissant d'un traité non-proportionnel (noté plus loin XL) d'une branche à déroulement long.

\section{LA MODELISATION DU PROBLEME}

\subsection{La distribution de sinistres}

\subsubsection{Les notations}

Le montant de sinistre total (aggregate claim amount) de l'XL est supposé suivre un processus composé de Poisson.

les notations sont les suivantes:

$P_{k}=$ probabilité de survenance de k sinistres à la charge de l'XL

$x=$ montant du sinistre individuel

$t=$ montant du sinistre total de l'XL

$F(x)=$ fonction de répartition de montant du sinistre individuel $x$

$f(x)=$ densité du montant du sinistre individuel $x$

$G(t)=$ fonction de répartition de la charge totale de l'XL.

Par définition

$$
\begin{aligned}
G(t) & =\sum_{k=1}^{\infty} P_{k} F^{* k}(x) & & x>0 \\
& =p_{0} & & x=0
\end{aligned}
$$

* désignant le produit de convolution.

1.1.2. La loi du nombre de sinistre

Dans l'étude qui suit, la loi du nombre de sinistres est une loi de Poisson. C'est 
une loi entrant dans le cadre de la famille de lois présentant des caractéristiques intéressantes pour un calcul simple de $G(t)$ par l'algorithme de Panjer (PANJER, 1981)

La valeur retenue dans la suite des calculs pour $\lambda$ est l'estimateur du maximum de vraisemblance de ce paramètre évalué sur les sinistres passés de la compagnie réassurée.

\subsubsection{La loi du montant du sinistre individuel}

Le montant du sinistre individuel est représenté par une loi de Pareto à un paramètre.

$$
\begin{aligned}
F(x) & =1-\left(\frac{x_{0}}{x}\right)^{\alpha} & & x \geqslant x_{0} \\
& =0 & & x<x_{0} .
\end{aligned}
$$

L'approximation de la distribution du montant du sinistre individuel par une loi de Pareto à un paramètre est le plus fréquemment utilisé par les actuaires dans le domaine de la réassurance. Elle présente un bon équilibre entre la simplicité des calculs et la qualité de l'ajustement.

De plus dans une famille plus vaste de distributions de sinistres utilisées en réassurance, elle offre d'une certaine manière, la sécurité la plus importante au réassureur (BENKTANDER, 1963).

Le $\alpha$ de Pareto peut être estimé sur les sinistres individuels passés de la compagnie réassurée ou sur un ensemble plus large (groupe de compagnies ou pays) suivant l'instabilité du portefeuille de la compagnie. L'estimateur convergent retenu est l'estimateur du maximum de vraisemblance (n étant le nombre de sinistres considérés)

$$
\hat{\alpha}=(n) / \sum_{1}^{n} \ln \left(x_{j} / x_{0}\right) .
$$

Afin de mettre en oeuvre la formule récurrente de l'algorithme de Panjer, la densité du sinistre individuel a été discrétisée en utilisant la méthode de l'ajustement des moments (PANJER et LUTEK, 1983). L'ajustement a été effectué sur l'espérance de la distribution de sinistre individuel.

Soit $h$ : longueur de l'intervalle (supposé constant) de discrétisation

La densité de probabilité associée à $F(x)$ sur un intervalle $\left[x_{0+i h}, x_{0+(i+1) h}\right]$ est répartie aux deux bornes de l'intervalle de la manière suivante:

Soit

$$
\begin{aligned}
x_{i} & =x_{0}+i h \\
x_{i+1} & =x_{0}+(i+1) h
\end{aligned}
$$

$f_{i}\left(x_{i}\right)$ densité de probalité de l'intervalle $\left[x_{i}, x_{i+1}\right]$ affectée au point $x_{i}$ 
$f_{i}\left(x_{i+1}\right)$ densité de probabilité de l'intervalle $\left[x_{i}, x_{i+1}\right]$ affectée au point $x_{i+1}$. Alors:

$$
\begin{aligned}
f_{i}\left(x_{i}\right) & =\int_{x_{i}}^{x_{i+1}}\left(h+x_{i}-x\right) / h \mathrm{~d} f(x) \\
f_{i}\left(x_{i+1}\right) & =\int_{x_{i}}^{x_{i+1}}\left(x-x_{i}\right) / h \mathrm{~d} f(x)
\end{aligned}
$$

\subsubsection{Application de l'algorithme de Panjer}

$$
\text { Si l'on note } \mathrm{g}_{j}=\operatorname{prob}[g(t)=j h] \text { et } \mathrm{f}_{i}=f_{i-1}\left(x_{i}\right)+f_{i}\left(x_{i}\right)
$$

l'algorithme de Panjer permet de calculer la fonction de répartition de sinistre total:

$$
\begin{aligned}
& g_{0}=\mathrm{e}^{-\lambda\left(1-p_{0}\right)} \\
& g_{j}=\frac{n}{J} \sum_{i=1}^{j} i f_{i} g_{j-i} \quad j=1,2, \ldots
\end{aligned}
$$

\subsection{La cadence de déroulement des sinistres}

Deux types de cadences interviennent: la cadence d'évaluation des sinistres (sur laquelle s'appuie le mécanisme de calcul du taux de prime glissant) et la cadence de réglement des sinistres à la charge de l'XL.

La cadence d'évaluation (de déclaration) des sinistres peut être soit:

Calculée année par année sur les données brutes du passé de la compagnie ou du marché étudié, (méthode de chain ladder sur données cumulées).

Ajustée sur une loi exponentielle négative avec une troncation après quelques années ( 5 ou 6 en général). Les paramètres de cette loi (de type $\mathrm{e}^{-\beta_{i} t^{\beta^{3}}}$ ) peuvent être estimés à l'aide d'une régression non linéaire et l'utilisation dans les calculs numériques d'un simple algorithme de Newton-Gauss.

La cadence de réglement des sinistres intervient au niveau d'une réduction sur taux de prime fixe afin de prendre en compte la période de temps s'écoulant entre le versement de la prime et le réglement des sinistres.

L'approche utilisée pour la cadence d'évaluation des sinistres peut être retenue. Dans le cas d'un ajustement sur une loi exponentielle négative, la troncation sera effectuée au bout d'une période plus longue (10 à 15 ans en général).

\subsection{Les produits financiers}

Le taux retenu correspond au taux de rendement que la compagnie de réassurance peut espérer sur la devise utilisée dans le traité. Si dans le cas d'une prime versée en une année, ce taux est concret, la compagnie pouvant investir cet argent dans 
un portefeuille de valeurs lui assurant une congruence avec les sorties (réglements de sinistres), en revanche pour un taux de prime glissant, l'on doit supposer l'invariabilité ou tout au moins une faible variation relative de ce taux pendant la période durant laquelle est versée la prime.

\subsection{Les chargements}

Ils peuvent être regroupés en deux types.

\section{Chargements 'commerciaux'}

Ils recouvrent les chargements pour frais de gestion, bénéfice, courtage. Ils seront pris égaux dans la suite de l'étude à un pourcentage de la sinistralité de l'XL.

\section{Chargements de fluctuation}

Ils prennent en compte l'influence du nombre de sinistres à charge de l'XL et de la portée de la tranche.

soit $x_{0}=$ priorité de l'XL

$k=$ plafond de l'XL/Priorité de l'XL

$\alpha=$ coefficient de la loi de Pareto

$\lambda=$ coefficient de la loi de Poisson

$\alpha$ étant toujours dans ce type de distribution supérieur à 1 .

$$
\begin{gathered}
\text { pour } \alpha=2 \quad \frac{\sigma}{E}=\left[\frac{2(\ln k-(1-1 / k))}{\lambda^{1 / 2}(1-1 / k)}\right]^{1 / 2} \\
\text { pour } \alpha>2 \quad \frac{\sigma}{E}=\frac{M_{2}^{1 / 2}}{\lambda^{1 / 2} M_{1}}
\end{gathered}
$$

avec

$$
\begin{aligned}
& M_{1}=\frac{x_{0}}{\alpha-1}\left[1-k^{1-\alpha}\right] \\
& M_{2}=2 x_{0}^{2}\left[\frac{k^{1-\alpha}-1}{\alpha-1}-\frac{k^{2-\alpha}-1}{\alpha-2}\right] .
\end{aligned}
$$

Généralement, le chargement de fluctuation adopté est un pourcentage de l'écart type de la distribution du sinistre total (BENKTANDER, 1975).

Dans l'application numérique qui suit ce chargement est pris égal à $20 \%$ de l'écart type. Ce pourcentage est utilisé assez fréquemment en réassurance (GERATHEWOHL et al., 1980).

Sans réelle justification théorique, un tel pourcentage permet de fixer le chargement de fluctuation à un niveau raisonnable ( $10 \%$ de la prime pure de l'XL) pour un XL réputé 'stable' $k \leqslant 2, \lambda \geqslant 5, \alpha=2$. 


\section{APPLICATION NUMERIQUE}

L'intérêt de la modélisation est de définir dans des cas concrets où l'on connain̂t la charge moyenne de l'XL, les bornes de taux de prime glissant.

L'on recherche un intervalle pour l'évolution du taux de prime glissant tel que l'on ait égalité entre la valeur actuelle (VA) du taux de prime fixe $t_{\mathrm{f}}$ et l'espérance de la valeur actuelle du taux de prime glissant $t_{\mathrm{g}}$ ( $d$ représentant la durée moyenne de réglement, $i$ le taux de rendement financier et $t$ le taux de chargement).

$$
\begin{gathered}
t_{\mathrm{f}}=\frac{\lambda x_{0}\left(1-k^{1-\alpha}\right)(1+i)^{-d}}{(1-t)(\alpha-1)} \\
t_{\mathrm{g}} \text { étant tel que } E\left(\operatorname{VA}\left(t_{\mathrm{g}}\right)=\operatorname{VA}\left(t_{\mathrm{f}}\right)\right.
\end{gathered}
$$

L'espérance de profit du réassurance est donc la même dans les deux cas.

Dans le cas d'un taux glissant, la variance du résultat pour le réassureur est certes réduite mais un tel type de taux entraîne des frais de gestion plus élevés (Clemeur, 1984). Cette augmentation des coûts couvre largement la baisse de fluctuation introduite par le taux glissant. Ainsi un profit équivalent pour le réassureur semble équitable aux deux parties, l'assureur bénéficiant d'un étalement dans le versement de ses primes de réassurance.

Dans les calculs qui suivent sont retenus des paramètres inspirés par la situation observée sur le marché français.

\section{HYPOTHĖSES DE CALCUL}

- priorité de la tranche

- plafond de la tranche

$: x_{0}$

- valeur du coefficient de Pareto

$: 4 x_{0}$

- cadence de déclaration des sinistres

$: 2$

- en fin d'année $0 \quad 20 \%$

- en fin d'année $1 \quad 55 \%$

- en fin d'année $2 \quad 85 \%$

- en fin d'année $3 \quad 95 \%$

- en fin d'année $4 \quad 100 \%$

- taux de rendement financier

$: i$

- durée moyenne de réglement des sinistres: 4,5 ans

- chargements de la prime glissante $\quad: 25 \%$

Les calculs ont été effectués avec le logiciel statistique SAS.

Le taux de prime minimum a été fixé à $60 \%$ du taux fixe afin d'obtenir une fourchette proche de celle rencontrée dans la pratique où le rapport entre taux maximum et taux minimum varie entre 2 et 3 .

Deux hypothèses de calcul ont été adoptées. La première considère que la prime

* Utiliser un tel type de cadence suppose implicitement que les sinistres une fois déclarés n'evoluent plus. En première approximation cette hypothèses sera retenue pour le marché français. 
fixe demandée n'intègre par de chargement de fluctuation. La seconde en revanche comprend un chargement de fluctuation tel qu-il est décrit en 1.4.

\subsection{Calcul avec taux de rendement financier fixe de $8 \%$}

La valeur du coefficient $\lambda$ varie de 1 à 10. Les deux graphiques présentent les résultats pour différentes valeurs de Lambda. Sur les graphiques le symbole 0

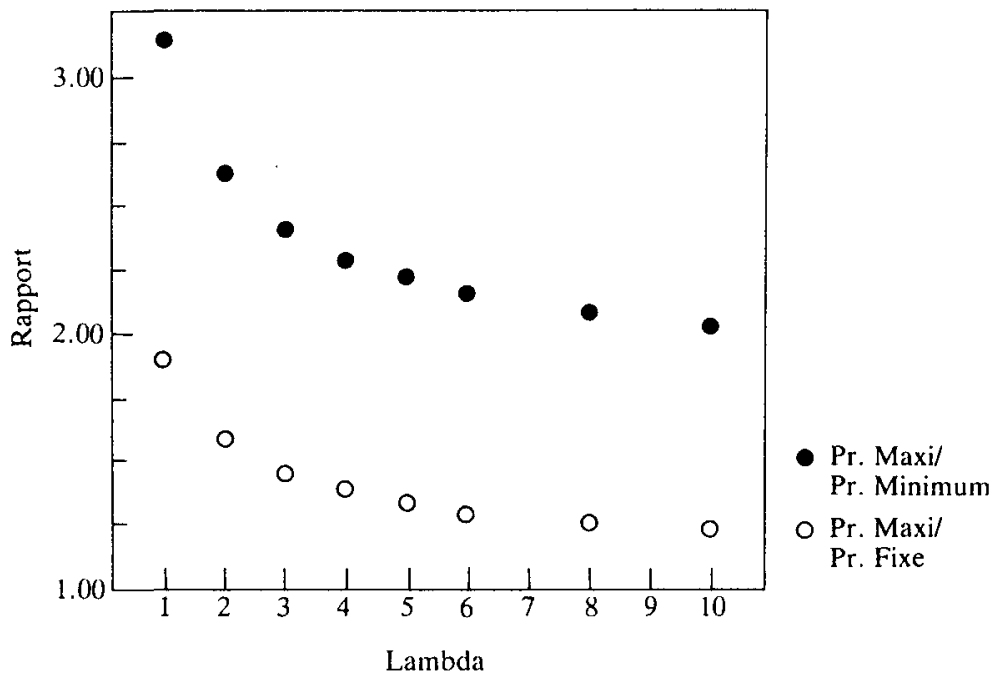

GraPhIQUE 1. Etude de primes glissantes pour differentes valeurs de lambda calcul de la prime avec uniquement un chargement proportionnel a l'esperance de la distribution

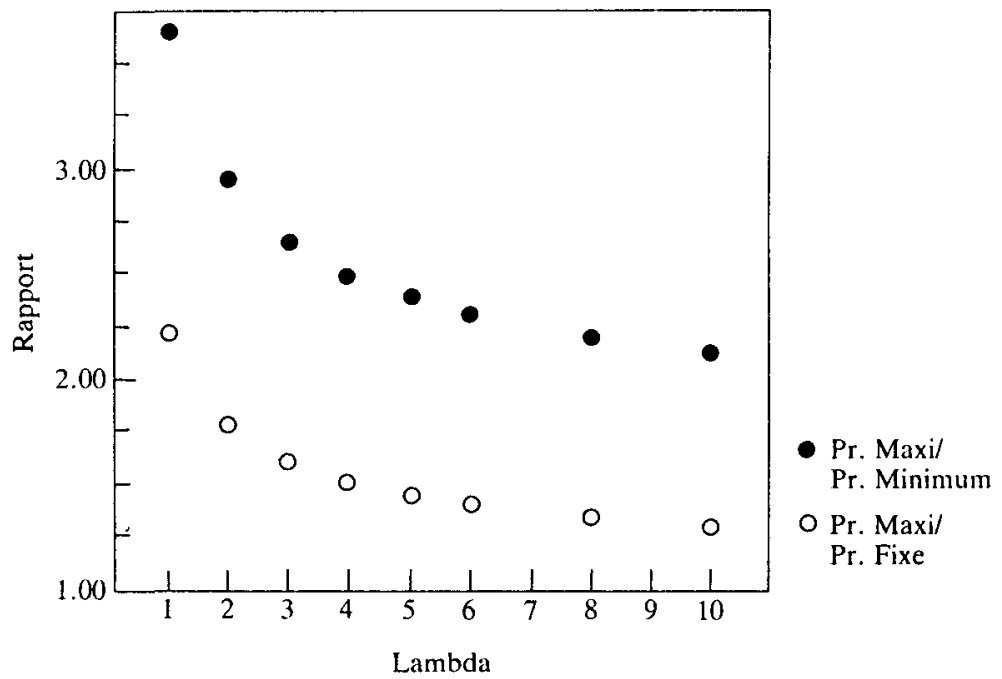

GRAPHiQUe 2. Etude de primes glissantes pour differentes valeurs de lambda calcul de la prime en incluant un chargement proportionnel a l'ecart-type de la distribution 
désigne le rapport prime maximum sur prime minimum tandis que le symbole + représente le rapport prime maximum sur prime fixe.

Sur les deux graphiques, dès que Lambda dépasse, 5 les variations enregistrées par les deux rapports deviennent rapidement très faibles.

L'écart, pour de petites valeurs de Lambda, entre la prime maximum et prime minimum est très important. Il en résulte que la prime minimum devrait être fixée à un niveau plus élevé que $60 \%$ de la prime fixe afin de dimineur l'étendue de la fourchette des taux.

\subsection{Calculs avec un taux d'intérêt variable}

Les variables de l'étendue de la fourchette de la prime glissante sont étudiées pour différentes valeurs du taux de rendement financier.

Les calculs sont effectués sur la base d'un Lambda égal à 5 .

Les deux hypothèses de détermination de la prime fixe sont conservées.

Dans le cas d'absence de rémunération financière et d'un taux fixe incluant des chargements de fluctuation, le taux maximum n'est pas atteint. Dans ce cas (hypothétique) 60\% du taux fixe comme taux minimum est un seuil trop bas. Il suffirait de remonter ce seuil afin d'obtenir un taux maximum et donc un taux glissant respectant l'égalité (9). Ainsi pour un taux minimum pris égal à $65 \%$ du taux fixe, le taux maximum obtenu représente 2,40 fois le taux fixe.

Le tableau ci-après présente les principaux résultats sous ces deux hypothèses:

TABLEAU 1

Etude de Primes Glissantes pour Differentes Valeurs du Taux de Rendement Financier

\begin{tabular}{|c|c|c|c|c|}
\hline \multirow[b]{2}{*}{$\begin{array}{l}\text { Taux de } \\
\text { rendement } \\
\text { financier }\end{array}$} & \multicolumn{2}{|c|}{$\begin{array}{l}\text { Taux fixe } \\
\text { avec chargements proportionnels } \\
\text { à l'esperance }\end{array}$} & \multicolumn{2}{|c|}{$\begin{array}{l}\text { Taux fixe } \\
\text { incluant des chargements } \\
\text { proportionnels à l'ecart type }\end{array}$} \\
\hline & $\frac{\text { Taux maximum }}{\text { Taux minimum }}$ & $\frac{\text { Taux maximum }}{\text { Taux fixe }}$ & $\frac{\text { Taux maximum }}{\text { Taux minimum }}$ & $\frac{\text { Taux maximum }}{\text { Taux fixe }}$ \\
\hline $0 \%$ & 2,66 & 1,60 & $\begin{array}{l}\text { Taux maxi } \\
\text { non-átteint }\end{array}$ & $\begin{array}{l}\text { Taux maxi } \\
\text { non-átteint }\end{array}$ \\
\hline $1 \%$ & 2,54 & 1,52 & 3,43 & 2,05 \\
\hline $2 \%$ & 2,45 & 1,47 & 3,13 & 1,88 \\
\hline $3 \%$ & 2,38 & 1,43 & 2,87 & 1,72 \\
\hline $4 \%$ & 2,32 & 1,40 & 2,71 & 1,62 \\
\hline $5 \%$ & 2,29 & 1,37 & 2,59 & 1,55 \\
\hline $6 \%$ & 2,25 & 1,35 & 2,51 & 1,50 \\
\hline $7 \%$ & 2,22 & 1,33 & 2,43 & 1,46 \\
\hline $8 \%$ & 2,20 & 1,32 & 2,39 & 1,43 \\
\hline
\end{tabular}

\section{CONCLUSION}

Une approche statistique pour l'appréciation des conditions proposées lors de l'offre d'une prime glissante sur un XL apparaît indispensable. C'est cette 
modélisation qui est adoptée dans notre société comme outil d'aide à la décision dans ce domaine.

L'auteur désire remercier les deux examinateurs anonymes de l'article ainsi que Monsieur Alois Gisler coediteur de l'ASTIN Bulletin pour leurs conseils et leurs judicieuses suggestions qui ont conduit à enrichir la première version de l'article.

\section{BIBLIOGRAPHIE}

BENKTANDER, Gunnar (1963), a note on the most dangerous and skewest class of distribution. ASTIN Bulletin 2 (1963) pp. 387-390.

BENKTANDER, Gunnar (1975), the Calculation of a fluctuation loading for an excess of loss cover. ASTIN Bulletin 8 (1975) pp. 272-278.

Clemeur, Hugo (1984) practical rating of variable accident excess-loss premiums. In Premium Calculation in Insurance Reidel Publishing Company, Dordrecht, Holland.

Gerathewolhl, Klaus et al. (1980) Reinsurance Principles and Practice Vol I p. 319, Verlag Versicherungswirtschaft e.V, Karlsruhe.

PANJER, Harry H. (1981), recursive evaluation of a family of compound distributions. ASTIN Bulletin 12 (1981) pp. 22-26.

Panjer, Harry H. et Lutek, B. W. (1983) Practical aspects of stop loss calculations. Insurance mathematics and Economics 2 (1983) pp. 159-117.

Charles LeVI

Compagnie Transcontinentale de Reassurance, 15 rue Louis le Grand, 75002

Paris, France. 